\title{
Developing experimental protocols for chronic irradiation studies: The application of a good practice guide framework
}

\author{
M.D. Wood ${ }^{1}$, J.L. Hingston ${ }^{1}$, D. Copplestone ${ }^{2}$ and I. Zinger $^{3}$ \\ ${ }^{1}$ School of Biological Sciences, University of Liverpool, Liverpool L69 3BX, UK \\ ${ }^{2}$ Environment Agency, Knutsford Road, Warrington WA4 1HG, UK \\ ${ }^{3}$ Swedish Radiation Protection Authority (SSI), 17116 Stockholm, Sweden
}

\begin{abstract}
A recent review of radiation effects data highlighted that information on the effects of low-dose, chronic exposure was, at best, fragmentary. These data are required to define the dose effect relationships, which must underpin assessments of the impact of ionising radiation on biota. In response, four Good Practice Guides (GPGs) have been produced as part of a protocol development framework that aims to harmonise experimental approaches, with a view to ensuring that appropriate endpoint data are collected and reliable dose effect relationships determined. The GPGs cover test species selection, endpoint selection, radiation exposure and experimental design considerations. A key guides researchers through the GPGs and the decisions made are recorded on a pro-forma, which is the basis of the experimental protocol. This paper describes the first application of the framework to a radiation effects study. The framework has been adopted by the UK Environment Agency as a document upon which future Agency-funded experimental work in this field will be based. It is hoped that the framework will gain acceptance in the wider scientific community and enable knowledge gaps to be addressed, in order that successful protection of non-human biota can be demonstrated.
\end{abstract}

\section{INTRODUCTION}

Statutory instruments and international agreements require that environmental protection from hazardous substances be demonstrated. Ionising radiation may not be identified specifically but, like chemical contamination, it poses a potential threat to the functioning of ecosystems. Proposed methods for demonstrating protection are commonly based around a generic scheme [1] in which the decision regarding the extent to which protection is being achieved is dependent upon two main components: (i) Estimation of doses to biota; (ii) Comparison with effects data and guidelines.

The availability of accurate data on the responses of non-human biota to ionising radiation is, therefore, an essential requirement of the impact assessment process. However, a recent review of effects data concluded that information on the effects of low-dose, chronic exposure is, at best, fragmentary [2]. This poses a difficulty for those charged with the responsibility of determining the extent to which adequate protection is being achieved. In response, the UK Environment Agency funded a Research \& Development project (P3-101/SP2), which aimed to facilitate research on the effects of radiation exposure on non-human biota. The project delivered a handbook [3], hereafter referred to as the SP2 handbook, containing four Good Practice Guides (GPGs), which together with a key and pro-forma, comprise the protocol development framework (Figure 1).

The SP2 handbook is designed to guide users through the decision-making process that underpins the development of research protocols for chronic irradiation studies on different groups of biota and provides advice on areas of research to be prioritised. It is anticipated that, given the extent of research required in this field, students could provide a valuable long-term resource for undertaking relevant experiments as Masters projects. For this reason, the SP2 handbook has been written to be accessible to both experienced radioecologists and those who are new to the field. 
The first protocols to be developed using the SP2 handbook were for a UK Environment Agency funded project (P3-101/SP7) on the effects of ionising radiation on soil fauna [4]. This paper demonstrates the application of the SP2 handbook to that study and highlights the key features of the protocol development process.

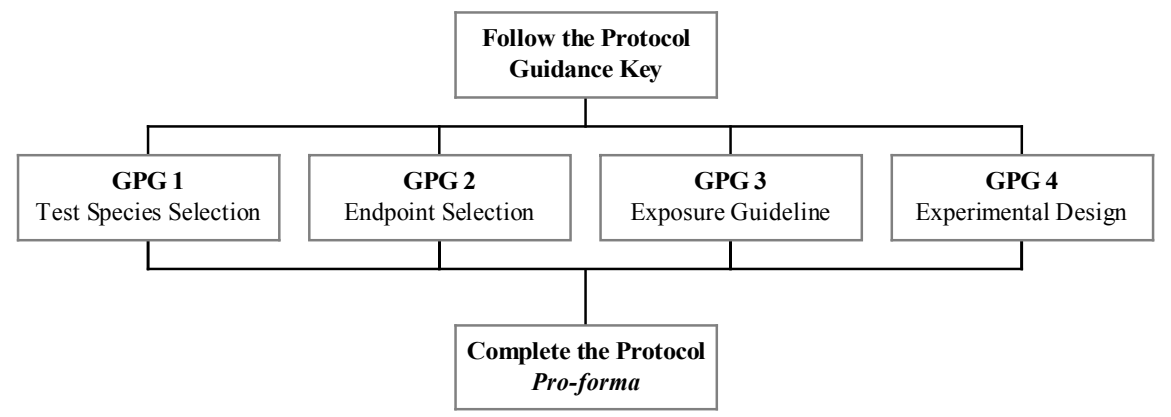

Figure 1. Overview of the main components of the protocol development framework.

\section{PROTOCOL DEVELOPMENT}

Researchers begin with a blank pro-forma and are directed to particular sections of the SP2 handbook by the key that drives the overall protocol development process. As researchers work through the key, all decisions and justifications are recorded on the pro-forma. The completed pro-forma is the basis for the protocols that are used in the final study. The following sections describe the completion of the pro-forma (Figure 2) for an investigation into the effects of ionising radiation on the woodlouse (Porcellio scaber), one of two test species studied in the P3-101/SP7 project.
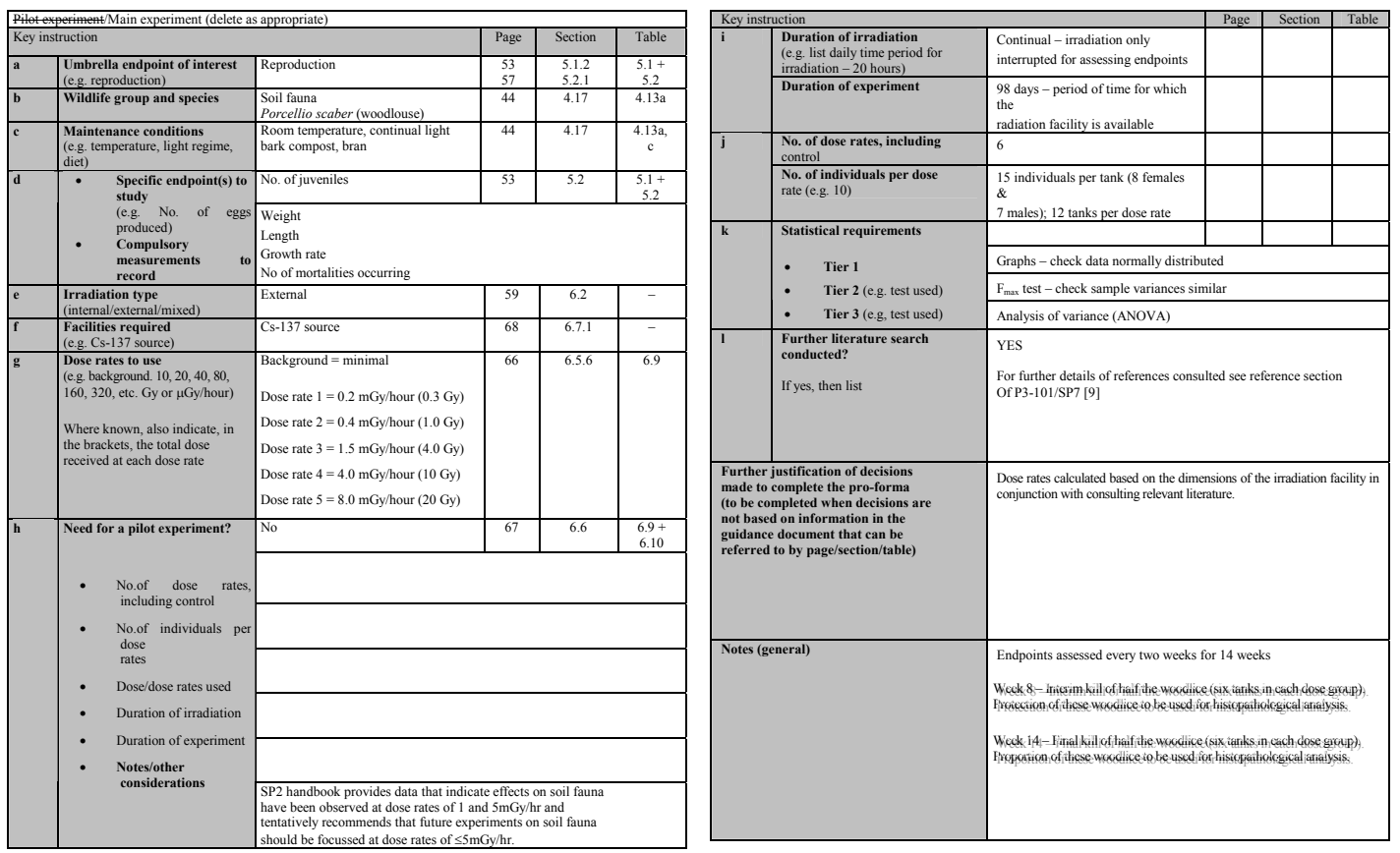

Figure 2. Completed pro-forma for a study on the effects of chronic radiation exposure on P. scaber [4]. 


\subsection{Test species selection (GPG1)}

GPG1 provides general advice on organism selection and highlights some of the factors that may influence the choice of test species (Table 1). Possible test species are categorised into thirteen wildlife groups and each group is discussed in a separate section of GPG1. All sections follow a common structure: discussion of the wildlife group's significance in terms of environmental protection; consideration of the availability of effects data for organisms within the group; evaluation of possible test species; consideration of husbandry and maintenance requirements; and recommendations of suitable test organisms to be used to represent the group. Tables are provided to demonstrate the range of species within each wildlife group that has been used in previous chronic irradiation studies and chemical toxicity tests. Where available, husbandry and maintenance information for recommended test species is provided as a series of reference tables.

The 'Soil fauna including microflora' wildlife group is of interest because of the tendency for radionuclides that are released into terrestrial ecosystems to become associated within the soil compartment and because of the key role that soil fauna play in energy and nutrient flow through processes such as decomposition. This group may therefore receive higher radiation doses than other organisms within the terrestrial environment. The main types of soil fauna are common to many terrestrial ecosystems. A comprehensive understanding of the effects of ionising radiation within this wildlife group is therefore applicable to a wide range of terrestrial environmental protection scenarios.

The woodlouse is one of three test organisms that the SP2 handbook recommends researchers use in radiation exposure studies on soil fauna. Woodlice are useful test organisms because they have few maintenance and space requirements and are easily kept in the laboratory. They have also been used successfully in both chronic irradiation and ecotoxicology studies and are a common component of many terrestrial ecosystems. P. scaber is particularly suitable for use because there is an established method for conducting reproductive endpoint studies on this species [5].

Once selected, the wildlife group and species to be studied should be recorded on the pro-forma along with any husbandry and maintenance information. Where the decision is based on information in the SP2 handbook, references to sources of relevant information (page number, section number, table number) should be noted. This allows the researcher to trace their decision-making process. Where the decisions are based on information from other sources, this should be recorded in the 'Further justification for decisions' section of the pro-forma.

Table 1. Some factors to consider when selecting species for laboratory studies.

\begin{tabular}{|c|c|}
\hline Economic importance & $\begin{array}{l}\text { Is this organism a food source for humans? } \\
\text { Does it have cultural value e.g. for recreation, sport or tourism? }\end{array}$ \\
\hline Ecological importance & $\begin{array}{l}\text { Is this organism a key species in the food chain? } \\
\text { Does it have a role in ecological processes e.g. carbon and nutrient cycling? }\end{array}$ \\
\hline Size & $\begin{array}{l}\text { Consider (i) laboratory space required; (ii) maintenance costs (e.g. of food); (iii) Quantity of } \\
\text { radionuclide required/source activity (cost, radiological safety) }\end{array}$ \\
\hline Breeding frequency & $\begin{array}{l}\text { Does the breeding frequency of the organism allow measurement of reproductive endpoints } \\
\text { e.g. continuous or periodic/annual breeding? }\end{array}$ \\
\hline Life-stage availability & $\begin{array}{l}\text { Early life-stages (seed, seedling, egg, embryo, larva) are often more radiosensitive than adults. } \\
\text { The ease with which these stages may be obtained and observed may be important in species choice. }\end{array}$ \\
\hline Husbandry & $\begin{array}{l}\text { Consider (i) How time-consuming is the maintenance required (e.g. frequency of cleaning)?; } \\
\text { (ii) Is it possible to achieve the environmental conditions required by the organism in a laboratory } \\
\text { environment (e.g. temperature, light)? ; (iii) Will the organism thrive in a laboratory environment? }\end{array}$ \\
\hline Information & $\begin{array}{l}\text { Is detailed information available on the use of the species in experiments (e.g. toxicology, genetics), } \\
\text { either from the literature or directly from other scientists? }\end{array}$ \\
\hline Availability & $\begin{array}{l}\text { Are good quality organisms available from suppliers or from the field, and what is known about the } \\
\text { variability of their responses? }\end{array}$ \\
\hline Cost & $\begin{array}{l}\text { Are the direct costs of the organisms and maintenance costs at a practical level (dependent on most } \\
\text { of the factors noted above)? }\end{array}$ \\
\hline
\end{tabular}




\subsection{Endpoint selection (GPG2)}

In order to assess the impact of a stressor (either chemical or radioactive) on a test species, it is necessary to determine the effect that different levels of exposure to that stressor have on particular endpoints. The key prompts researchers to decide on one of four umbrella endpoints ${ }^{1}$ (morbidity, mortality, mutation and reproduction [1]) to focus on, but highlights the importance or reproduction studies for environmental protection purposes. It is generally accepted that assessing this umbrella endpoint is important, when investigating the impacts of ionising radiation on biota, because successful environmental protection requires the maintenance of overall ecosystem function and biodiversity. This is inherently linked to success, at the population level, of organisms occupying the different niches within an ecosystem. Therefore, any reduction in reproductive success, or reduction in fitness that is passed to the progeny as a result of genetic mutation in the germ cells, may impact on ecosystem function. Many current impact assessment approaches use the population as the unit of protection rather than the individual; hence reproduction is the most ecologically relevant endpoint to consider when collecting effects data to underpin assessments.

The choice of reproduction specific endpoint $(s)^{2}$ to measure will depend on the test species selected, the particular interests of the researcher and the facilities available. The SP2 handbook cannot, and does not attempt to, provide advice on all possible specific endpoints, but it does highlight some of the commonly-measured specific endpoints and the wildlife groups to which they can be applied. It is recommended that researchers conduct a literature search to identify the most appropriate endpoints to measure for their chosen test species. For $P$. scaber, the principal specific endpoint selected was number of viable juveniles produced, but a literature search also revealed previous studies that have investigated histopathological alterations in the hepatopancreas of woodlice as a result of exposure to cadmium and zinc $[6,7]$. To investigate whether similar effects may be caused by radiation exposure, histopathological assessment of the hepatopancreas was included in the list of specific endpoints to be assessed in P3-101/SP7. Once selected, specific endpoints should be recorded in the appropriate section of the pro-forma.

The pro-forma also lists some specific endpoint measurements that all researchers are encouraged to observe and record. These include changes in physical appearance of organisms (e.g. length and weight), number of mortalities during the experiment and measurements of growth rate. These endpoints are proposed because subtle changes to specific endpoints that appear of little relevance to reproductive success in a laboratory study may still significantly affect the fitness of an organism in the wild. This is particularly true in the animal kingdom where many sexually reproducing organisms actively select their mate. For example, under laboratory conditions, a bird whose plumage has been changed may still successfully breed because there is a limited pool of individuals available. In the wild, however, the affected individual may be unsuccessful at breeding. It should be noted, therefore, that the SP2 handbook suggests a cautious approach when applying laboratory-derived data to field scenarios. It also highlights the need for researchers to be vigilant in their approach to radiation effects studies and, in particular, to ensure that they record any effects on other endpoints as a matter of course. However, it should be noted that the SP2 handbook is not meant to dictate the endpoints to be studied but guide the decision-making process.

\subsection{Exposure guideline (GPG3)}

This GPG aims to familiarise researchers with the issues surrounding the use of ionising radiation in laboratory studies and to detail the different approaches that can be adopted for exposing organisms to particular dose rates. The SP2 handbook is primarily targeted towards conducting studies on the effects of chronic radiation exposure so accurate dosimetry is essential. Details of methods for conducting both external and internal dosimetry are presented in GPG3.

To produce a dose-effect curve, the radiation dose rates studied must span the threshold at which an effect is observed. To assist researchers in identifying the threshold dose, GPG3 includes tables for

\footnotetext{
1 A descriptive term used to group biological effects of particular types, e.g. morbidity, mortality, mutation and reproduction.

2 Quantifiable characteristics of an organism, or its progeny, that can be used to investigate the effects of a contaminant on a particular umbrella endpoint.
} 
each wildlife group that list observed effects at chronic dose rates (Table 2).

No data were available for woodlice specifically but information on other soil fauna suggested that doses in the range of $1 \mathrm{mGyh}^{-1}$ to $5 \mathrm{mGyh}^{-1}$ may result in some observable effects. The six dose rates selected for the P.scaber study (listed in Figure 1) cover this range and extend up to 8mGyh ${ }^{-1}$. The $8 \mathrm{mGyh}^{-1}$ upper dose rate was selected with the aim of ensuring that the dose-effect threshold was spanned for at least one of the specific endpoints being monitored.

Table 2. Effects observed in soil fauna and insects following chronic radiation exposure.

\begin{tabular}{|c|c|c|c|c|}
\hline \multirow{6}{*}{\begin{tabular}{|l}
$\begin{array}{l}\text { Dose Rate } \\
(\mu \mathbf{G y} / \mathbf{h}) \\
<100\end{array}$ \\
$<10$
\end{tabular}} & Species & \multicolumn{2}{|c|}{ Radiation Description } & \multirow{4}{*}{$\begin{array}{l}\text { Endpoint } \\
\text { Mortality } \\
\text { Reproduction } \\
\text { Morbidity } \\
\text { Morbidity }\end{array}$} \\
\hline & Earthworm & alpha & Reduced numbers compared with control plots. & \\
\hline & & & Smaller, reproductive and histological changes & \\
\hline & Insect larvae & alpha & Reduced numbers compared with control plots & \\
\hline & Scorpion & gamma & Increased chromosomal aberrations & Mutation \\
\hline & Midge & Mixed & Increase in chromosome aberrations & Mutation \\
\hline $100-1000$ & & & No data available & \\
\hline$(1-5) \times 10^{3}$ & Earthworm & & Reduced population size & Mortality \\
\hline$(5-10) \times 10^{3}$ & $\begin{array}{l}\text { Myriapods } \\
\text { Spiders }\end{array}$ & beta & Reduced numbers compared with control plots & Mortality \\
\hline \multirow[t]{2}{*}{$(10-50) \times 10^{3}$} & $\begin{array}{l}\text { Earthworm } \\
\text { Bark beetle }\end{array}$ & & Reduced pupal survival & Morbidity \\
\hline & $\begin{array}{l}\text { Soil } \\
\text { invertebrates }\end{array}$ & & Reduced population sizes & Mortality \\
\hline$>50 \times 10^{3}$ & Ants & & Behavioural changes of colony & Morbidity \\
\hline
\end{tabular}

\subsection{Experimental design (GPG4)}

Careful experimental design is essential if the results obtained are to be reproducible and scientifically robust. In particular, consideration should be given to the data required to test the central hypothesis of the study with a given degree of confidence. The SP2 handbook highlights the need for researchers to identify, prior to undertaking experimental work, the methods of data analysis to be used. Many statistical tests have a range of assumptions and requirements associated with them. These may include the independence of samples ${ }^{3}$, the distribution of the data, the minimum number of categories (e.g. dose rates) and the minimum numbers of replicates. The assumptions of a test must be valid if the researcher is to have confidence in the results obtained.

To ensure that the overall experimental protocol meets the requirements of the statistical test selected, researchers should consider the analysis of experimental data as a three-tiered system (Figure 3 ). Within the SP2 Handbook, researchers are asked to identify the data analysis approach they will adopt and the tasks to be undertaken at each assessment tier. This can then be used to evaluate their experimental approach with regard to the data requirements of each tier. The researcher can revise their protocol as a result of this evaluation, prior to investing resources on experimental work that will not allow their hypothesis to be tested successfully.

For the project P3-101/SP7, Analysis of Variance (ANOVA) was selected as the Tier 3 method so that the results could be compared to identify any significant differences. The ANOVA test has a number of requirements, for example that the observations should be normally distributed and have similar sample variances. Tier 1 of the data analysis was to inspect the data graphically to confirm that they were normally distributed, Tier 2 included an $\mathrm{F}_{\max }$ test to determine whether or not the variances of the samples were similar and Tier 3 was the ANOVA test.

A review of the proposed experimental protocol revealed that the expected data outputs would

\footnotetext{
${ }^{3}$ Within a sample, the value of any one observation is not inherently linked to the value of any other observation.
} 
satisfy the requirements of each tier of the data analysis process. The completed pro-forma was therefore accepted as the experimental protocol to be used in the study of the effects of chronic radiation exposure on $P$. scaber.

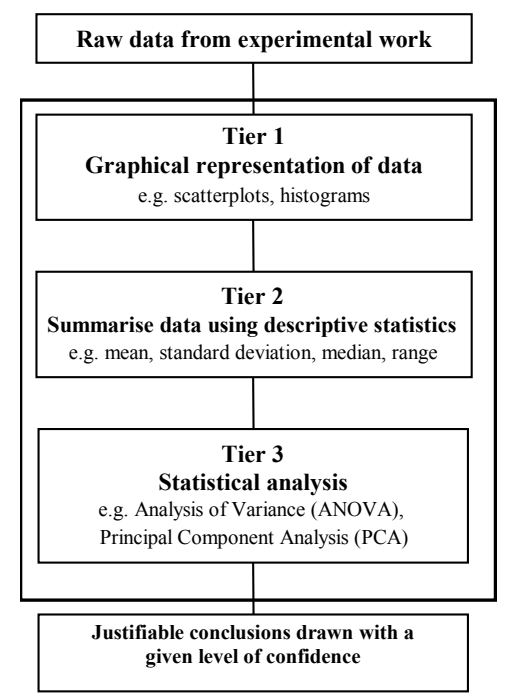

Figure 3. The three-tiered approach to data analysis.

\section{SUMMARY}

The SP2 handbook provides a protocol development framework for designing experiments on the effects of ionising radiation on biota. The framework is applicable to acute and chronic exposure studies but the emphasis is placed on the latter, particularly in relation to reproductive effects, because these are the data that are required to underpin current and developing methodologies for assessing the impact of ionising radiation on biota. The SP2 handbook highlights areas of research to prioritise but is not prescriptive and should always be used in conjunction with other literature. It handbook has been adopted by the UK Environment Agency as a document upon which future Agency-funded experimental work on the effects of chronic, low-level exposure to ionising radiation will be based. It is hoped that the framework will gain acceptance in the wider scientific community and enable knowledge gaps to be addressed, in order that protection of biota can be demonstrated.

\section{Acknowledgments}

The authors are grateful to the UK Environment Agency for funding this work.

\section{References}

[1] FASSET Deliverable 6, C-M Larsson., C. Jones, J. M. Gomez-Ros and I. Zinger Eds. (FASSET, 2004) pp. 1-74.

[2] FASSET Deliverable 4, D. Woodhead and I. Zinger Eds. (FASSET, 2003) pp. 1-196.

[3] Wood M. D., Knowles J. F., Whittaker J., Copplestone D., Malcolm H. M. and Bielby S., Developing experimental protocols for chronic irradiation studies on wildlife R\&D Technical Report P3-101/SP2, (Environment Agency, Bristol, 2003) pp. 1-96.

[4] Hingston J. L., Knowles J. F., Walker P. J., Wood M. D., and Copplestone D., "Effects of ionising radiation on soil fauna" R\&D Technical Report P3-101/SP7, (Environment Agency, Bristol, 2004) pp. 1-64.

[5] Spurgeon D., Svendsen C., Hankard P., Weeks J., Kille P. and Fishwick S., "Review of sublethal ecotoxicological tests for measuring harm in terrestrial ecosystems" R\&D Technical Report P5-063/TR1, (Environment Agency, Bristol, 2002) pp.1-192.

[6] Odendaal J. P. and Reinecke A. J., Ecotox. and Environ. Safety 56 (2) (2003) 319-325.

[7] Znidarsic N., Strus J. and Drobne D., Environ. Tox. and Pharm. 13 (3) (2003) 161-174. 